\title{
Abstracts of the meeting of the Clinical Genetics Society held at the University of Wales College of Medicine, Cardiff, on 18 and 19 April 1986
}

\author{
Osteogenesis imperfecta type IIA: evidence for dominant \\ inheritance \\ ID YOUNG* ${ }^{*}$ E M THOMPSON $\dagger$, C M HALL $\ddagger$, AND M E PEMBREY $\dagger$ \\ ${ }^{*}$ Department of Child Health, Leicester Royal Infirmary, \\ Leicester; †Genetics Unit, Institute of Child Health, Lon- \\ don; and $¥$ Department of Radiology, Hospital for Sick \\ Children, London.
}

During a United Kingdom study of lethal osteogenesis imperfecta congenita, 30 cases were ascertained which showed radiological changes typical of type IIA, with minimal skull mineralisation, broad, short, unmodelled femora and humeri, angled tibiae, flat vertebral bodies, and broad continuously beaded ribs. The sex ratio did not differ significantly from 1 . All of the cases were isolated. There were 19 foreborn and 19 afterborn sibs, all healthy. Only one set of parents, of Asian origin, was consanguineous. Mean paternal age was $34 \cdot 19 \pm 7 \cdot 17$ years, which differed significantly $(p<0.01)$ from the general population mean paternal age. These observations are in keeping with published accounts of heterozygosity for an abnormal type I collagen allele and suggest that most cases of type IIA osteogenesis imperfecta are likely to result from new dominant mutations.

Recurrence risks and prognosis in severe sporadic osteogenesis imperfecta with fractures at birth E M THOMPSON* ${ }^{*}$, I YOUNG $\dagger$, C M HALL $\ddagger$, AND M E PEMBREY ${ }^{*}$ Mothercare Unit of Paediatric Genetics, Institute of Child Health, London WC1; tLeicester Royal Infirmary, Leicester; and $¥$ The Hospital for Sick Children, Great Ormond Street, London WC1.

The clinical application of the Sillence classification of osteogenesis imperfecta (OI) is limited by genetic heterogeneity in type III and overlap between types II and III and between III and IV, especially when the patient is assessed in the neonatal period. We conducted a UK study of severe sporadic OI presenting with fractures at birth, in order to determine recurrence risks. Ninety-eight probands who met these criteria survived the perinatal period; they correspond to the overlap group of Sillence type III/sporadic type IV. Of their 142 sibs, eight were affected, giving a maximum recurrence risk of $5 \cdot 6 \%$. This is likely to be an overestimate (to be discussed); the estimated recurrence risk lies between 4.5 and $5.6 \%$. There were seven families with more than one affected child (six with two affected and one with three affected). In a separate study of 57 probands with perinatally lethal OI
(Sillence type II), 30 had type IIA, with no recurrences (see previous abstract by Young et al). Fourteen probands were classified as type IIB: in these 14 families, there was a single recurrence in one and two recurrences in another, in total three affected sibs out of 17 . Three probands had type IIC OI with three normal sibs. In 10 families in which the proband was unclassifiable since the $x$-ray could not be personally reviewed, there were no recurrences in 22 sibs. No patient who survived the perinatal period had the type IIA radiological appearance at birth, indicating a bad prognosis for this type. There is a gradient of radiological severity at birth which correlates with mortality, ranging from the most severe (type IIA) to the mild (type I fresh dominant mutation). The intermediate group of IIB and 'severe survivors' show overlap in clinical and radiological appearance, so that it may be more appropriate to consider the overall recurrence risk of $\mathbf{7 \%}$ for this group. It is likely that a small, as yet indistinguishable, subgroup of these are autosomal recessive, the majority arising as fresh dominant mutations.

Peripheral neurofibromatosis: guidelines for counselling based on a population study in South Wales

$S$ M HUSON, D A S COMPSTON, AND P S HARPER

Section of Medical Genetics, University of Wales College of Medicine, Cardiff.

A population based study of peripheral neurofibromatosis (NF) has been performed in South Glamorgan and West Gwent (population 667900 ). A total of 136 people with NF was alive in the study area on 1 August 1985, giving a minimal prevalence of $1 / 4900$. The affected subjects came from 70 families in which no clinically normal obligate gene carriers were identified. For 49 persons there was no history of NF in preceding generations but in only 42 of these could this be confirmed by examination of or accurate history concerning their parents. The mutation rate of NF is therefore $3.36 \times 10^{-5}$ if all 49 represent new mutations, but only $3.14 \times 10^{-5}$ if $n=42$. In those cases aged $>5$ years for whom information was available $(n=124), 19 \%$ had been or were in a remedial class with $10 \%$ attending a special school, although only one person was severely retarded. Considering all family members alive aged $>18$ years and their dead relatives, the incidence of malignancy (including CNS tumours) related to NF was approximately $6 \%$. Other major complications of NF in the study population were plexiform neurofibromas $39 \%$ ( $8 \%$ causing severe cosmetic problems) and other neurological problems $10 \%$. For counselling 
purposes the complications of NF can be considered in three groups: those which (1) occur during childhood and cause lifelong morbidity (large plexiform neurofibromas, orthopaedic problems, and intellectual handicap), combined incidence $19 \%$; (2) occur at any time but are 'treatable' (neurological, renal artery stenosis, visceral and endocrine tumours), combined incidence $6 \%$; and (3) malignancy $6 \%$. As many of the complications of NF occur in childhood, affected children should be particularly closely monitored, but none of the complications occur trequently enough to warrant regular screening investigations.

\section{Generalised collagen deficiency in patients with mitral valve prolapse}

ANNE H CHILD, M BURLEIGH, G LEECH, AND A LEATHAM

Department of Histopathology, St George's Hospital Medical School, London SWI7 ORE.

In a detailed study of 90 adult patients with mitral valve prolapse (MVP), 65/90 (72\%) showed signs of connective tissue weakness in two or more systems (ocular, musculoskeletal, dermatological, pulmonary) other than the cardiovascular system. Of the 38 patients who had aortic compliance measured, $15(39 \%)$ had significantly raised values indicating weakness of the aortic wall. Nine of 22 clinic patients and 10 of 20 operated patients (total $45 \%$ ) were found to have abnormal skin collagen ratios, indicating probable systemic collagen deficiency as a basis for the mitral valve prolapse. Eight of the 20 patients who came to operation had low collagen type III levels in skin and all suffered rupture of chordae tendineae. This evidence suggests that the majority of patients $(72 \%)$ with MVP of any degree of severity have an underlying, genetically determined abnormality of collagen biosynthesis and that the most severely affected have a generalised deficiency of type III collagen. Protection of the aortic root by propranolol in Marfan
syndrome

R E PYERITZ

Johns Hopkins University, School of Medicine, Baltimore, USA.

Aortic regurgitation and dissection account for most early deaths in Marfan syndrome and usually occur when the aortic root becomes dilated. Because aortic root enlargement is progressive and probably due to the repeated strain of ventricular ejection, chronic reduction in inotropy and chronotropy might retard dilatation and prevent complications. To test these hypotheses, 70 patients were randomly assigned to propranolol treatment or to no drug treatment, their aortic root dimensions measured at least annually, and their outcomes documented. Both treatment and control groups were analysed based on whether physical growth was complete. Groups did not differ as to age, sex, initial aortic root dimension, or length of follow up (average 6.5 years). Propranolol dose was optimised to reduce inotropy by about $30 \%$ and to prevent the post- exercise heart rate from exceeding 100 . The rate of change of aortic diameter was slower in both children (1.4 $\mathrm{mm} /$ year vs 2.2$)$ and adults $(0.5$ vs 1.4$)$ who took propranolol $(p<0 \cdot 02)$. In the treated group, four patients (including the two non-compliers) required aortic root surgery, whereas in the control group one died of dissection, one died of arrhythmia, and six required surgery (including two with acute dissection) $(p<0.05)$. These results suggest that chronic beta-adrenergic blockade reduces the rate of aortic dilatation and the development of severe complications in Marfan syndrome.

Data on the linkage between DNA probes and the cystic fibrosis locus

R WILLIAMSON AND THE CYSTIC FIBROSIS GENETICS RESEARCH GROUP

Department of Biochemistry, St Mary's Hospital Medical School, London W2 1PG.

After the initial demonstration that CF shows linkage to the polymorphic enzyme system paroxonase, several groups demonstrated linkage to polymorphic DNA markers: TCRB (St Mary's), 917 (Toronto), Epo (Integrated Genetics), 3.11 (St Mary's), met (Salt Lake City), 7C22 (St Mary's), and COL1A2 (St Mary's). These have been ordered, and met and 3.11 are both approximately $1 \mathrm{cM}$ from CF, with a lod score approaching 70 or more. Other informative probes are also being studied and the majority of families with a living CF member are informative for carrier exclusion or prenatal diagnosis with an accuracy of approximately $96 \%$ at a confidence of 0.95 . Attempts to move from linkage to mutation involve a combination of pulse field gel elecrophoresis, microdissection directly from chromosomes, the use of open reading frame vectors and cosmid overlap libraries, and the development of direct functional assays to identify genes which code for membrane proteins which determine ion transport. Because the existing probes are informative in many families and closely linked to the CF locus, it has also been possible to offer prenatal diagnosis to several pregnancies at risk in the first trimester.

Combined use of RFLPs and microvillar enzyme assay in the prenatal diagnosis of cystic fibrosis

D J H BROCK

Human Genetics Unit, Western General Hospital, Edinburgh EH4 $2 X U$.

The availability of the tightly linked DNA probes, pJ3-11 and met, has made possible the first trimester prenatal diagnosis of cystic fibrosis (CF) on CVS samples. Recombination frequencies between the $C F$ gene and these probes are sufficiently low to make DNA based diagnosis the method of choice in informative couples. RFLP analysis fails when there is no living affected child (or conserved tissues) to establish phase, or where both parents are non-informative. About $40 \%$ of pregnancies at risk for CF may fall into these categories. In such cases the couple will continue to be referred for amniocentesis and 
mid-trimester microvillar enzyme assay. A more difficult situation occurs when a couple is only partially informative for RFLP analysis. In such cases an uninformative CVS sample raises the risk of CF to 1 in 2 . If such a pregnancy is then referred for microvillar enzyme assay, a negative test will still leave a residual risk of $\mathrm{CF}$ of 5 to $10 \%$. If a couple is particularly anxious to avoid recurrence of a CF child, careful consideration must be given as to whether such a residual risk is satisfactory after two separate diagnostic procedures.

Structural rearrangements in the parents of children with primary trisomy 21

D A COUZIN, J L WATT, AND G S STEPHEN

Department of Genetics, University of Aberdeen, Foresterhill, Aberdeen AB9 $2 Z D$.

A retrospective analysis of the cytogenetic studies carried out on the parents of children with regular trisomy 21 Down's syndrome was undertaken. In a total of 128 parents referred routinely to our laboratory after the birth of their affected child, three structural abnormalities $(\operatorname{inv}(4)(\mathrm{p} 14 \mathrm{q} 23), \operatorname{inv}(\mathrm{X})(\mathrm{p} 22 \mathrm{q} 11), \mathrm{t}(1 ; 13)(\mathrm{q} 23 ; \mathrm{q} 22))$ not involving chromosome 21 were detected. This is about 10 times the frequency expected based on current figures from consecutive newborn studies. This supports the contention made by others that an interchromosomal effect does exist in man. It is suggested that centres who routinely analyse the parents of their trisomy 21 referrals in an unbiased fashion should review their records. In addition to its undoubted scientific value, such data should prove useful in the genetic counselling of carriers of structural rearrangements.

\section{Genetic aspects of early childhood scoliosis}

D GOUDIE, J L TOLMIE, B YEUNG, A N CONNER, R A C CONNOR, AND J M CONNOR

University Department of Medical Genetics, Duncan Guthrie Institute of Medical Genetics, Yorkhill, Glasgow.

Ninety-six families with early onset scoliosis were evaluated. These were divided into three groups: resolving idiopathic scoliosis (15 families), progressive infantile idiopathic scoliosis (21 families), and congenital scoliosis (60 families). The children with congenital scoliosis were subdivided into those with neural arch defects and those with other vertebral malformations. One family with congenital scoliosis was considered to have autosomal recessive spondylothoracic dysplasia, but otherwise the recurrence risk for scoliosis was low in each group studied. There was, however, an increased risk (3.5\% for sibs) for neural tube defects in the families with congenital scoliosis (with or without neural arch defects in the proband). This sib risk was apparent for probands with only a single hemivertebra in addition to probands with multiple vertebral anomalies. This recurrence risk and epidemiological similarities would support the conclusion that neural tube defects and other vertebral malformations are aetiologically related.
Further data confirming apparent prevention of orofacial clefts by periconceptional supplementation with vitamins and folic acid

MARIE TOLAROVÁ

Institute of Experimental Medicine, Czechoslovak Academy of Sciences, Lidových milicí 61-63, 12000 Prague 2, Czechoslovakia.

Since 1982, when the first results of our method of primary prevention were published in the Lancet, we have continued this study. There are now 250 pregnancies which have ended after periconceptional supplementation with vitamins and folic acid. The results confirm our previous findings and correspond well with a four threshold model of liability to cleft lip and palate, which has been suggested in our previous studies. The best results were found in the subgroup of male probands with unilateral cleft and no positive influence of prevention was found in the subgroup of female probands with a bilateral defect. According to our model, the first subgroup with a lower value of heritability has a higher proportion of exogenous factors in the aetiology, which might be influenced by supplementation, in contrast to the last subgroup, where no effect of supplementation was found.

Prenatal assessment of haemophilia A using DNA probes S MALCOLM, E ROBERTSON, K HARPER, J FISHER, E GOLDMAN, C $H$ RODECK, R $S$ MIBASHAN, AND M E PEMBREY Institute of Child Health, and King's College Hospital, London.

Twenty-two pregnant women ( 23 pregnancies) at risk of bearing a haemophilic son were referred for genetic diagnosis. Five were obligate carriers and another five had clearly abnormal coagulation. The linked DNA probes DX13 and St14 and the gene specific (exon 17-18) factor VIII:C probe were used to search for restriction fragment length polymorphisms in the women and available family members. Of the 12 women with uncertain carrier status, three were excluded using the gene specific probe and one was shown to have a very low risk combining DX13 with coagulation results. In three of the 22 women, RFLP analysis of the family samples was uninformative using probes available at that time. In the remaining 16 women informative for prenatal diagnosis (17 pregnancies), two fetuses were female, five were unaffected males (two by the VIII:C probe, one by DX13, and one on St14 analysis), and two were affected males by DX13 probing. Seven pregnancies had other outcomes; two were unsustained, one woman did not proceed with diagnosis, three yielded unsatisfactory specimens, and one result is still awaited.

Urgent carrier testing for Duchenne muscular dystrophy using pERT87 probes

J FISHER, M E ROBERTSON, S MALCOLM, AND M E PEMBREY Institute of Child Health, Guilford Street, London.

We have been offering a service since August 1985 to clarify carrier status in women with a family history of 
Duchenne muscular dystrophy, combining information from the pedigree, creatine kinase levels, and the pERT87 probe, and more recently $\mathrm{XJ} 1 \cdot 1$ (using a recombination fraction of 0.05 for calculations). Analysis on 42 women has been completed and in 35 their carrier status clarified. Seven of the women were referred during the first trimester of pregnancy. In four of these seven women, carrier risk was lowered to a point where they no longer wanted fetal sexing planned on the basis of earlier carrier risks of 8.5 to $33 \%$. One women was shown to have a $>90 \%$ carrier risk but chose to continue the pregnancy without prenatal tests. Carrier state could not be clarified using probes in two women. As these women were heterozygous it was possible to identify an allele carrying high risk and an allele with low risk $(<0 \cdot 6 \%)$ and so prenatal exclusion was offered.

Emery-Dreifuss muscular dystrophy: linkage to polymorphic DNA probes located on the terminal long arm of the $X$ chromosome

N S T THOMAS* ${ }^{*}$, H WILLIAMS ${ }^{*}$, L J ELSAS $\dagger$, L C HOPKINS $\ddagger$, AND P S HARPER ${ }^{*}$

${ }^{*}$ Section of Medical Genetics, University of Wales College of Medicine, Cardiff; and Departments of TPediatrics and $\ddagger$ Neurology, Emory University School of Medicine, Atlanta, Georgia, USA.

As part of our continuing interest in the inheritance of $X$ linked muscular dystrophies we have undertaken a linkage study of Emery-Dreifuss muscular dystrophy (EMD) families to ascertain whether this disorder is genetically linked to the Xp21 region already known to contain the loci for both Becker and Duchenne muscular dystrophies, or whether, as recently suggested by Hodgson et al (CGS meeting November 1985), it is located on the terminal long arm. Using a panel of polymorphic DNA probes covering the whole of the $\mathrm{X}$ chromosome, we are currently analysing a large four generation family with $X$ linked Emery-Dreifuss muscular dystrophy previously reported by Hopkins et al (Ann Neurol 1981; 10: 230-7). We have DNA samples from more than 100 persons, including 18 affected males. DNA probe DX13 (DXS15) is shown to be linked to the EMD locus in this family; with two recombinants from 17 informative meioses, LIPED gives a maximum lod score of 2.87 at $10 \mathrm{cM}$. The probe for factor VIII has shown no recombinants in 12 meioses (maximum lod score 4.25). No linkage was found with the short arm probes 754 and pERT87.

\footnotetext{
Minisatellite DNA determination of zygosity in twins discordant for sacral agenesis

M D'A CRAWFORD, J CHESHIRE, AND T M WILSON

Northwick Park Hospital, Harrow, Middlesex.
}

Sacral agenesis is usually sporadic, but reports of occasional familial occurrence raise the possibility that genetic factors may be involved in its aetiology. There have been no twin reports. Adult twin brothers are presented who are discordant for sacral agenesis. The proband has partial sacral agenesis, unilateral ureteric duplication, corrected hypospadias, and relative short stature. Blood group and HLA markers gave identical phenotypes and yielded a probability of monozygosity of $99.93 \%$. This was confirmed with the single stranded minisatellite DNA probes $\lambda 33.6$ and $\lambda 33.15$, kindly provided by Alec Jeffries, which hybridised to identical DNA fragments in Southern blots from the twins. The demonstration that twins discordant for sacral agenesis are monozygotic is evidence for an environmental aetiology in this instance. It is possible that monozygotic twinning may itself have predisposed to the malformation, or that a common pathogenetic mechanism may have led to both the twinning and the malformation.

\section{Segregation and risks in reciprocal translocation carriers: a computer programme \\ MIGUEL A DE ARCE* , PATRICK M GRACE $\dagger$, AND \\ SUSAN P McMANUS* \\ *Department of Pathology, Children's Hospital, Dublin 1; and + Faculty of Engineering, University College, Dublin 2.}

This is a programme for IBM-PC written in Basic. Given the breakpoints in a reciprocal translocation carrier (rcp) the programme calculates the imbalance in the 16 gametes that could be produced, as listed in ISCN $1972-300$ bands. The 16 gametes are then listed in order of increasing imbalance, which is measured as a percentage of the haploid autosomal length occurring in trisomy or monosomy. Once the least imbalanced gamete (LIGA) has been calculated, its imbalances are compared with the results of a literature search spanning 10 years and listing the maximum imbalances found in partial (or total) aneuploids for each chromosome arm. Two hypotheses were tested; (1) that the aneuploid offspring is always produced by the least imbalanced gamete, and (2) that for every chromosome there is a size of imbalance beyond which only miscarriages occur. The first hypothesis was verified in $90 \%$ of 210 family histories of rcp carriers with aneuploid live births. The second hypothesis could not be verified in more than $50 \%$ of rcp with histories of recurrent miscarriages. Risks of (a) aneuploid live births, (b) miscarriage, (c) phenotypically normal live births, and (d) stillbirths are estimated in the following way. (1) Given a problem rcp, the programme selects from the memory the histories of rcp carriers that produced offspring imbalanced for the same chromosome arm as the LIGA of the problem carrier. (2) Within those, a further selection is made of those with the same mode of segregation and sex of carrier parent. (3) Weinberg's correction for ascertainment bias is automatically made. (4) Homogeneity tests for paternal and maternal carriers are performed, and histories pooled if justified. Risks are estimated as frequencies from the histories so selected.

A microcomputer register for Huntington's chorea M SARFARAZI, G WOLAK, AND O QUARRELL Section of Medical Genetics, University of Wales College of Medicine, Cardiff.

We have written a package for the entry and manipulation 
of family history and other data specifically for Huntington's chorea. The programme is written in dBASE III and is currently running on an IBM PC (XT) microcomputer. Persons or branches of a pedigree can easily be added, edited, listed, or deleted and the programme keeps track of the relationships between persons. The programme is menu driven and user friendly. There are several full screen options which are each designed for a given task. This is the first in a series of programmes and further versions will improve in efficiency and the number of functions available. The package is completely selfcontained and requires no detailed user intervention. Pedigrees of any size can be drawn and the system controls all the necessary manual processes for the maintenance of a clinical register. There is a recall/visit facility for user defined risk groups in selected geographical areas. The ability to record all data without dependence on a mainframe computer is an important aid to maintaining confidentiality.

\section{Linkage of a polymorphic marker for the type III collagen gene (COL3A1) to autosomal dominant Ehlers-Danlos syndrome type IV in a large Belgian pedigree}

A DE PAEPE*, A NICHOLLS $\dagger$, P NARICISI $\dagger$, R DALGLEISH $\ddagger$, F DE KEYSER* ${ }^{*}$, M MATTON*, AND F M POPE $\dagger$

*Department of Genetics, University of Gent, Belgium; †Dermatology Research Group, Clinical Research Centre, Harrow, and $¥$ Department of Genetics, University of Leicester.

Ehlers-Danlos syndrome type IV is a clinically and genetically heterogeneous disease in which premature rupture of arteries has been associated with type III collagen abnorıalities. We have investigated a large, three generation Belgian family in which 11 members had a form of autosomal dominant EDS IV. Type III collagen measured in skin and cells cultured from skin and blood vessels from three affected subjects was approximately $75 \%$ of control compared to $10 \%$ in the acrogeric form of EDS IV. The type III collagen genes in this family were examined by restriction enzyme mapping using a cDNA clone from the $3^{\prime}$ end of the gene. The family was informative for a polymorphic variation associated with the enzyme AvaII (Dalgleish et al. Nucleic Acids Res 1985;13:4609). The low frequency allele segregated with the disease indicating that the type III collagen gene is the disease locus in this family. The association of this polymorphism with the disease will permit prenatal diagnosis, if required, in a situation where direct protein analysis might give ambiguous results. The polymorphism will also act as a marker in gene cloning experiments designed to identify the precise mutation.

Prenatal exclusion in a family where choroideremia is associated with a small $\mathrm{Xq}$ deletion

M E ROBERTSON, S V HODGSON, J A FISHER, S MALCOLM,

B JAY, C FFAR, M BOBROW, AND M E PEMBREY

Paediatric Research Unit, Guy's Hospital, and Institutes of Ophthalmology and Child Health, London.
Choroideremia is an $\mathrm{X}$ linked progressive degeneration of the choroid and retina leading to blindness in the 50s and not usually associated with mental retardation. Female carriers show characteristic retinal changes of irregular pigmentary disturbances. Nussbaum et al (1985) reported close linkage between the choroideremia locus (TCD) and the probe DXYS1 $(90 \%$ confidence limits $\theta=0-9 \mathrm{cM})$. This probe detects an invariant band on the $Y$ and polymorphic bands on the $\mathrm{X}$. The consultand, who had the fundoscopic changes of choroideremia, presented for counselling at 9 weeks' gestation. Her affected brother was noted to have mild mental retardation. DNA from family members and a chorionic villus sample were analysed using DXYS1. The affected brother showed a clear Y specific band but no $\mathrm{X}$ band, indicating that the probe region on the $\mathrm{X}$ had been deleted. The fetus showed a $\mathrm{Y}$ specific band and a clear $\mathrm{X}$ band, indicating it had not inherited the deletion. Subsequent chromosome analysis revealed a complex rearrangement with a pericentric inversion and a deletion of $\mathrm{Xq} 13 \cdot 1 \rightarrow \mathrm{q} 21 \cdot 2$ in the consultand and her affected brother.

\section{Clinical presentation of mosaic tetrasomy $12 p$}

D DONNAI, C M WHITE, O STECKO, D HUNTFR, AND T ANDREWS Department of Medical Genetics, St Mary's Hospital, Manchester.

A female infant with many dysmorphic features was referred for genetic evaluation at 3 weeks of age. Chromosome analysis of cultured lymphocytes had revealed a normal female karyotype $(46, \mathrm{XX})$ in all 30 cells examined. Her phenotype strongly suggested a diagnosis of mosaic $12 p$ tetrasomy and a skin biopsy was arranged. Chromosome analysis of cultured fibroblasts revealed an extra metacentric chromosome in $74 \%$ of cells, compatible with an isochromosome of $12 \mathrm{p}$. Confirmatory enzyme studies for LDH-B are in progress. Since the abnormal cell line is rarely detected in cultured lymphocytes it is the typical phenotype which should alert the physician to the diagnosis. Affected babies have flat facies with a wide glabella region, puffy eyelids, and short palpebral fissures. The nose is short and the philtrum long and unusually prominent. There is abnormal scalp hair patterning with deficiency of frontal hair. The limbs tend to be shortened and joint contractures are common. Other structural abnormalities are occasionally seen and all reported children have had profound psychomotor retardation.

Adult polycystic disease and the $\alpha$ globin locus: a genetic linkage study in South Wales

L LAZAROU, F DAVIES, S REEDERS*, G COLES $\dagger$, M SARFARAZI, AND P S HARPER

Section of Medical Genetics, University of Wales College of Medicine, Cardiff; *John Radcliffe Hospital, Headington, Oxford; and †Renal Unit, Cardiff Royal Infirmary, Cardiff.

Following the detection of close linkage between the adult polycystic kidney disease (APKD) and $\alpha$ globin loci 
(Reeders et al, 1985), we have studied South Wales families in order to confirm the linkage and assess the possibility of genetic heterogeneity. A total of 127 kindreds is known in South Wales, of which nine have been studied for genetic linkage. The probes used to detect polymorphisms are those for $\alpha$ globin and for the associated 3-HVR sequence. Results for the four kindreds completed so far give a provisional lod score of $4 \cdot 0$ at 0 recombination.

Duchenne muscular dystrophy, adrenal hypoplasia, glycerol kinase deficiency, and mental retardation associated with an Xp21 interstitial deletion

ANGUS CLARKE, S H ROBERTS*, N S THOMAS, AND J WILLIAMS $\dagger$ Section of Medical Genetics and *Child Health Laboratories, University of Wales College of Medicine, Cardiff; and tDepartment of Biochemistry, Princess of Wales Hospital, Bridgend.

A number of boys with DMD, adrenal hypoplasia, glycerol kinase deficiency, and mental retardation associated with an Xp21 chromosomal deletion have been described. We have identified a similar case from his history of DMD, severe mental retardation, and an Addisonian disorder. Cytogenetic analysis revealed an interstitial deletion of the short arm of the X chromosome, from Xp21·1 $\rightarrow$ p22 11 , representing about $9 \%$ of the length of the $X$ chromosome. His mother was hemizygous for the deletion but his sister, who presented in early pregnancy, had two normal $\mathrm{X}$ chromosomes. DNA probe analysis confirmed a deletion in the proband, as probes 754, pERT87-1, and C7 consistently failed to hybridise to his DNA. His sister was heterozygous for the RFLP associated with 754, thus confirming she had two normal X chromosomes. We were therefore able to reassure this girl that prenatal diagnosis was unnecessary. There was no evidence of chronic granulomatous disease or retinitis pigmentosa in this case. Biochemical studies revealed gross glyceroluria and hyperglycerolaemia, indicating glycerol kinase deficiency. We are currently investigating glycerol levels in urines from other Duchenne patients.

Hypohidrotic ectodermal dysplasia: localisation to the proximal long arm of the $X$ chromosome

A CLARKE, M SARFARAZI, AND N THOMAS

Section of Medical Genetics, University of Wales College of Medicine, Cardiff.

A linkage study of 18 families with hypohidrotic (anhidro- tic) ectodermal dysplasia has been performed. The previously suggested linkage to DXYS1 has been confirmed, and linkage to probes 58-1 and 19-2 has been established (see table). Analysis of one multiply informative kindred has demonstrated recombination for DXYS1 but not for $19-2$ at one meiosis, and recombination for 19-2 but not for DXYS1 at another meiosis. This strongly suggests that the HED locus is flanked by DXYS1 proximally and 19-2 distally on the long arm of the $\mathrm{X}$ chromosome.

$\begin{array}{lll}\text { Probe } & \theta_{\max } & \text { Lod score } \\ \text { 58-1 } & 0.07 & 3.6 \\ \text { DXYS1 } & 0.03 & 5.8 \\ 19-2 & 0.09 & 4.3\end{array}$

Clinical study of these and other families suggests a high mortality in early childhood, but we have found no evidence of mental retardation as a primary feature of the condition. Carrier detection is often possible on the basis of dental examination, and whole back sweat tests are useful to supplement this when necessary.

Assignment of the gene for dyskeratosis congenita to Xq28 D GATHERER, F C GRAY, L A PIRRIT, N AFFARA, AND J M CONNOR

University Department of Medical Genetics, Duncan Guthrie Institute of Medical Genetics, Glasgow.

Dyskeratosis congenita is an $\mathrm{X}$ linked recessive disorder with diagnostic dermatological features, bone marrow hypofunction, and a predisposition to neoplasia in early adult life. Linkage analysis was undertaken in an extensive family with the condition using the $\mathrm{Xg}$ blood group and 17 cloned $\mathrm{X}$ chromosomal DNA sequences which recognise restriction fragment length polymorphisms (RFLPs). No recombination was observed between the locus for dyskeratosis congenita $(D K C)$ and the RFLPs identified by DXS52 (St14-1) $(\mathrm{Zmax}=3.33$ at $8 \max =0$ with $95 \%$ confidence limits of 0 to $14 \mathrm{cM}$ ). Similarly no recombination was observed for the disease locus and F8 $(\mathrm{Zmax}=1.23$ at $8 \mathrm{max}=0)$ nor for DXS15 (DX13) $(\mathrm{Zmax}=1.62$ at $8 \mathrm{max}=0)$, but both of these markers were only informative in part of the family whereas $D X S 52$ was fully informative. $D X S 52, D X S 15$, and $F 8$ are known to be tightly linked and have previously been assigned to $\mathrm{Xq} 28$. Thus the gene for dyskeratosis congenita can be assigned to $\mathrm{Xq} 28$. These DNA sequence polymorphisms will be of clinical value for carrier detection and prenatal diagnosis. 